Supporting information

for

\title{
Inhibition of the Oxygen-sensing Asparaginyl Hydroxylase Factor Inhibiting Hypoxia- inducible Factor (FIH): A Potential Hypoxia Response Modulating Strategy
}

Yue Wu, ${ }^{a}$ Zhihong Li, ${ }^{a}$ Michael A. McDonough, ${ }^{b}$ Christopher J. Schofield,,${ }^{b}{ }^{*}$ and Xiaojin Zhanga, ${ }^{\mathrm{a}}{ }^{*}$

a Jiangsu Key Laboratory of Drug Design and Optimization, and Department

of Chemistry, China Pharmaceutical University, Nanjing 211198, China

b Chemistry Research Laboratory, Department of Chemistry and the Ineos Institute for Antimicrobial Research, University of Oxford, 12 Mansfield Road, Oxford OX1 3TA, United Kingdom

Corresponding authors

E-mail addresses: zxj@cpu.edu.cn (X. Zhang), or christopher.schofield@chem.ox.ac.uk (C. J. Schofield).

\section{Contents}

I. Figure S1. Crystallographic analysis of an ASPP2 fragment in complex with FIH .S2 


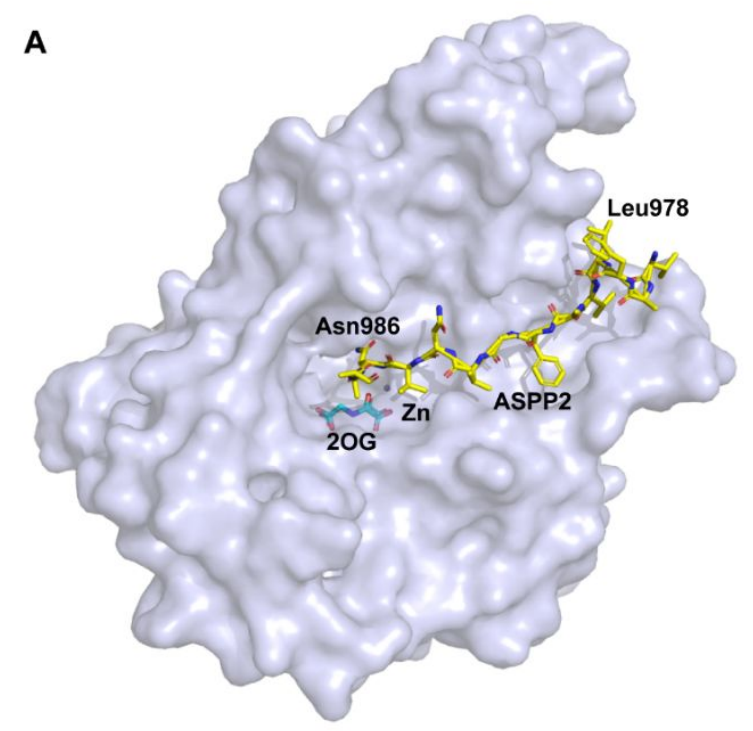

B

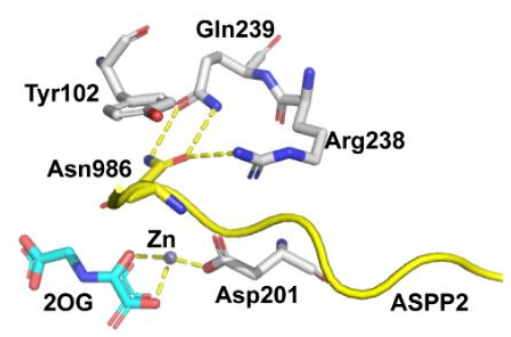

C

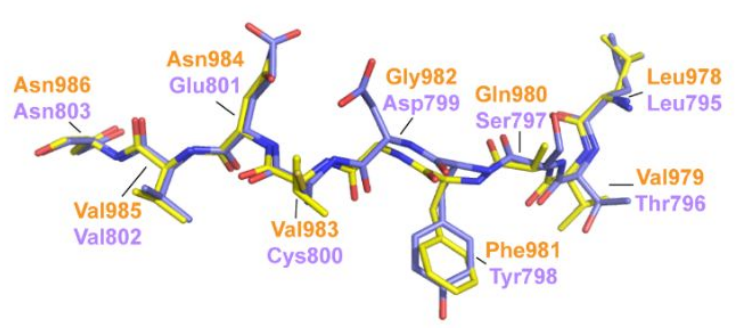

D

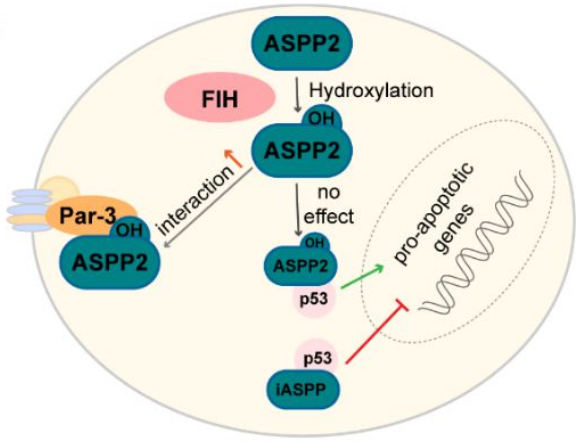

Figure S1. Crystallographic analysis of an ASPP2 fragment in complex with FIH. A) View from an FIH-ASPP2 complex structure (PDB ID: 6HKP). ASPP2 fragments are shown as sticks in yellow above a van der Waals surface of FIH. ASPP2 Asn986 is buried at the active site and is located close to the active site metal ion ( $\mathrm{Zn}$ substituting for Fe); B) Interactions between ASPP2 Asn986 and FIH. Asn986 is located between Tyr102 of FIH and the metal ion. It is positioned to form intermolecular hydrogen bonds with Asp201, Arg238, and Gln239; C) Stick representation of superimposed peptide fragments of ASPP2 (978-986, yellow) and HIF- $\alpha$ CTAD (795-803, purple) bound to FIH; D) Schematic showing proposed impacts of FIH on ASPP2 protein interactions. Par-3 is important in cell-polarization. 